DOI: 10.4274/jarem.galenos.2020.3630

J Acad Res Med 2020;10(3):298-302

\title{
A Rare Presentation of Retinoblastoma as a Fungating Orbital Mass: A Case Report
}

\author{
Kenan Kıbıcı1, (1) Berrin Erok22, (1) Onat Akın3 \\ ${ }^{1}$ Altınbaş University, Bahçelievler Medical Park Hospital, Clinic of Neurosurgery, İstanbul, Turkey \\ ${ }^{2}$ Cihanbeyli State Hospital, Clinic of Radiology, Konya, Turkey \\ ${ }^{3}$ Patomed Laboratory, Department of Pathology, İstanbul, Turkey
}

Cite this article as: Kıbıcı K, Erok B, Akın O. A Rare Presentation of Retinoblastoma as a Fungating Orbital Mass: A Case Report. J Acad Res Med 2020;10(3):298-302

\begin{abstract}
Retinoblastoma (RBL) arises from the precursor cells of the retinal neuroepithelium. It is the most common primary malignant intraocular tumor in children. Primary orbital RBL is an extraocular disease detected clinically or radiologically at the time of diagnosis. It is a direct metastasis of an intraocular RBL. It very rarely presents as an exuberant fungating orbital mass, like in the present case, and is found in more advanced, untreated cases. Magnetic resonance imaging (MRI) is the preferred imaging modality for the evaluation of the tumor, vital orbital structures such as the optic nerve, and intracranial involvements. Computed tomography (CT) is useful in the evaluation of adjacent bony structures and calcifications. Herein, we report the case of a 3-year-old boy who presented to our clinic with a large, rapidly growing cauliflower-like mass protruding out of the left orbit and invading the surrounding soft tissues. MRI showed a $10 \times 8 \times 7.5 \mathrm{~cm}^{3}$ infiltrative heterogeneously enhancing left orbital mass with some necrotic and hemorrhagic components. Metastatic leptomeningeal enhancements were widespread in the intracranial region. CT revealed erosive destructive changes at the lateral orbital wall. Following left orbital enucleation, the diagnosis of RBL was made by histopathological examination, which showed sporadic Flexner-Wintersteiner type rosette formations. The patient underwent surgery and thereafter received radiotherapy and adjuvant chemotherapy. No recurrence was observed after 5 years. Although rare, orbital extension of RBL is one of the major contributors to mortality. Early diagnosis and detailed radiological evaluation are necessary to establish intracranial involvement and distant metastasis at the time of diagnosis.
\end{abstract}

Keywords: Primary orbital retinoblastoma, Flexner-Wintersteiner rosette, magnetic resonance imaging (MRI), leptomeningeal metastasis

\section{INTRODUCION}

Various neoplasms can arise from orbital structures. Retinoblastoma (RBL) is a neoplasm that arises from the retina, affecting the precursor cells of the retinal neuroepithelium. It is the most common primary malignant intraocular tumor in children. It is exclusively found in young children, and most cases were diagnosed before the age of five (1). RBL may be sporadic or secondary to a germline mutation associated with the inactivation of the RBL protein tumor suppressor gene, which is usually inherited. The hereditary form, which constitutes approximately half of the cases, tends to occur bilaterally at younger age $(2,3)$. Affected children are also at increased risk of developing trilateral RBL (unilateral orbital RBL and pineoblastoma) and osteosarcoma (4). Sporadic disease forms usually occur in older children and are typically solitary (1). Primary orbital RBL is diagnosed as an extraocular disease and a direct extension of intraocular RBL. It is

ORCID IDs of the authors: K.K. 0000-0002-5912-9648; B.E. 0000-0001-8036-547X; O.A. 0000-0002-9878-8726. 
very rarely reported in developed countries due to early diagnosis and treatment. However, in developing and underdeveloped countries, extraocular extension of RBL is not an unusual presentation and is one of the major factors of poor prognosis. It is different from secondary orbital RBL, which is defined as orbital recurrence several weeks to years after enucleation for intraocular RBL. The most frequent manifestations are leukocoria (a white pupil), similar to that in intraocular RBL or proptosis (5). In more advanced and untreated cases, massive extraocular tumor spread very rarely results in a fungating giant orbital mass, as in the present case. Diagnosis is made by histopathological examination. In patients with extraocular extension of $\mathrm{RBL}$, radiological imaging is important to determine the exact size and extent of the tumor. Herein, we report a case of a primary orbital RBL with intracranial involvement at the time of diagnosis in a 3-year-old boy.

\section{CASE PRESENTATION}

In August 2013, an otherwise healthy 3-year-old boy presented to our clinic with a giant, rapidly enlarging cauliflower-like mass protruding out of the left orbit and invading the surrounding soft tissues (Figure 1). He recalled that the lesion appeared approximately 6 months earlier and started to grow rapidly for the last 3 months. On physical examination, no regional lymph node enlargement or organomegaly was detected. Magnetic resonance imaging (MRI) of the orbit showed a $10 \times 8 \times 7.5 \mathrm{~cm}^{3}$ solid infiltrative left orbital lesion obliterating the superior orbital fissure. The globe and retro-orbital structures lost their normal morphology. The left optic nerve (ON) was indistinguishable (Figure 2). There were some areas of necrosis on T2-weighted images (T2-WI) (Figure 2) and some linear hemorrhagic components on T1-WI (Figure 3). The mass extended into the left $\mathrm{ON}$ through the orbital apex into the optic chiasm, which was occupied by a nodular component of the mass (Figure 4). Following contrast administration, non-necrotic areas of the mass showed moderate to marked enhancement (Figure 5). In the intracranial region, enhanced metastatic meningeal lesions

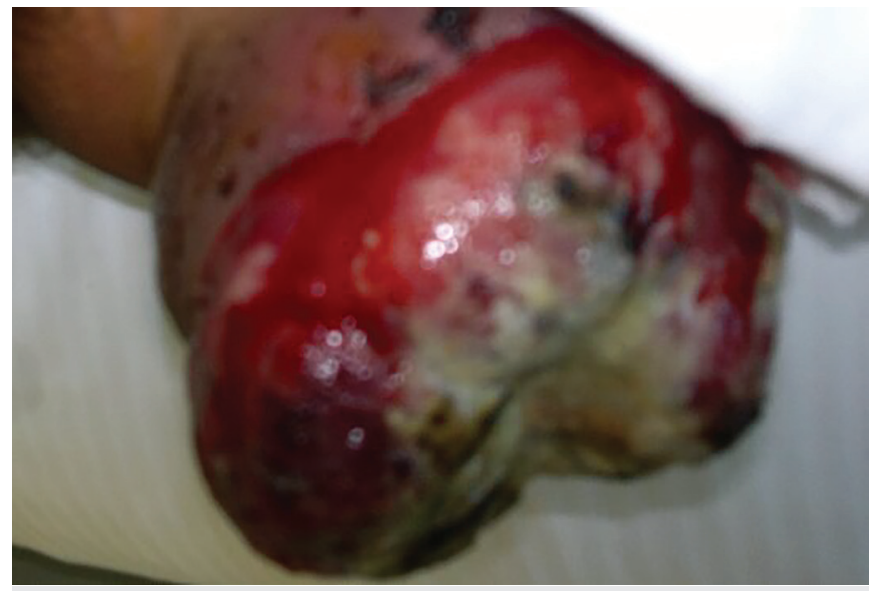

Figure 1. A large, cauliflower-like mass protruding out of the left orbit and invading the surrounding soft tissues in a 3-yearold boy

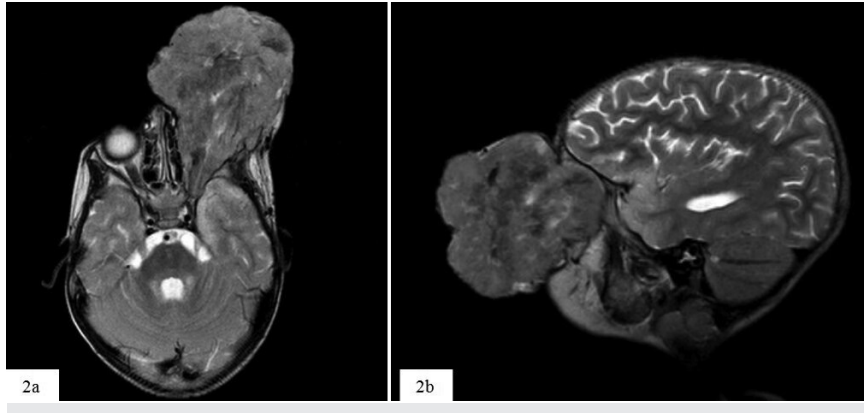

Figure 2. (a) Axial and (b) sagittal T2-weighted magnetic resonance images of the left orbit showing exophytic left orbital mass, which is heterogeneously isointense to the cortical gray matter with some areas of necrosis. The superior orbital fissure is obliterated, and the left optic nerve is indistinguishable

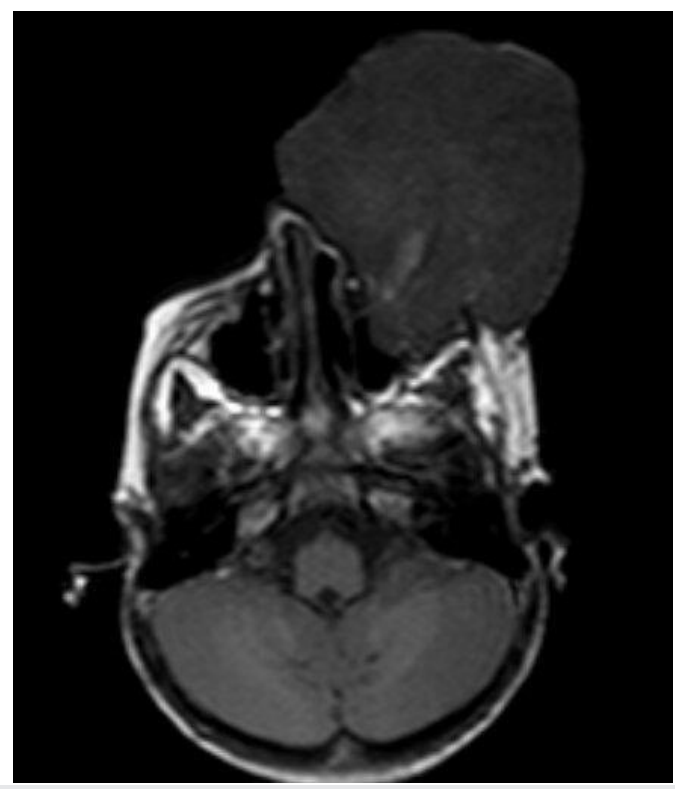

Figure 3. Axial T1-weighted magnetic resonance image showing some linear hyperintense hemorrhagic components

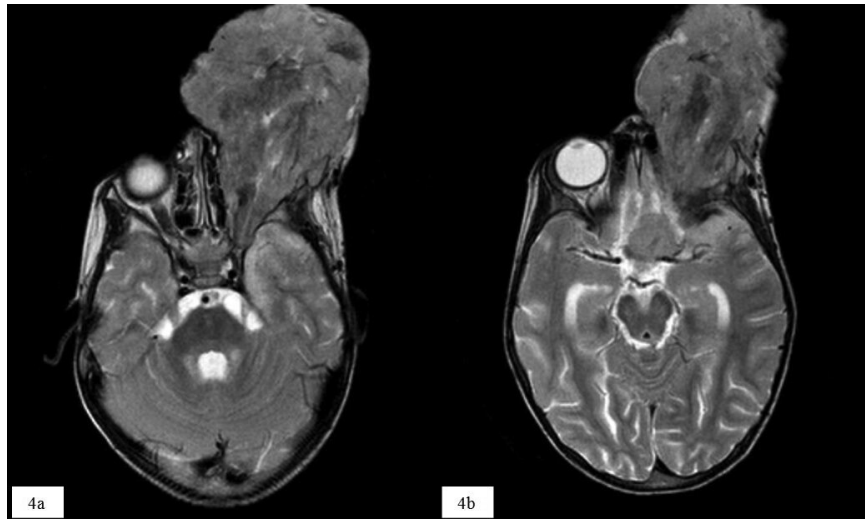

Figure 4. Axial T2-weighted magnetic resonance image showing (a) the extension of the mass into the left optic nerve through the orbital apex into the optic chiasm and (b) a nodular component of the mass occupying the optic chiasm 
were detected in both supratentorial and infratentorial regions. The lesions were the widest at the left middle cranial fossa on the temporal lobe (Figure 6). On computed tomography (CT), the lesion was isodense compared with the vitreous, and there were erosive destructive changes at the lateral orbital wall, with no extension into the maxillary and ethmoid sinuses. There were some hemorrhagic foci, but no calcification was detected (Figure 7). Further systemic evaluation revealed neither systemic

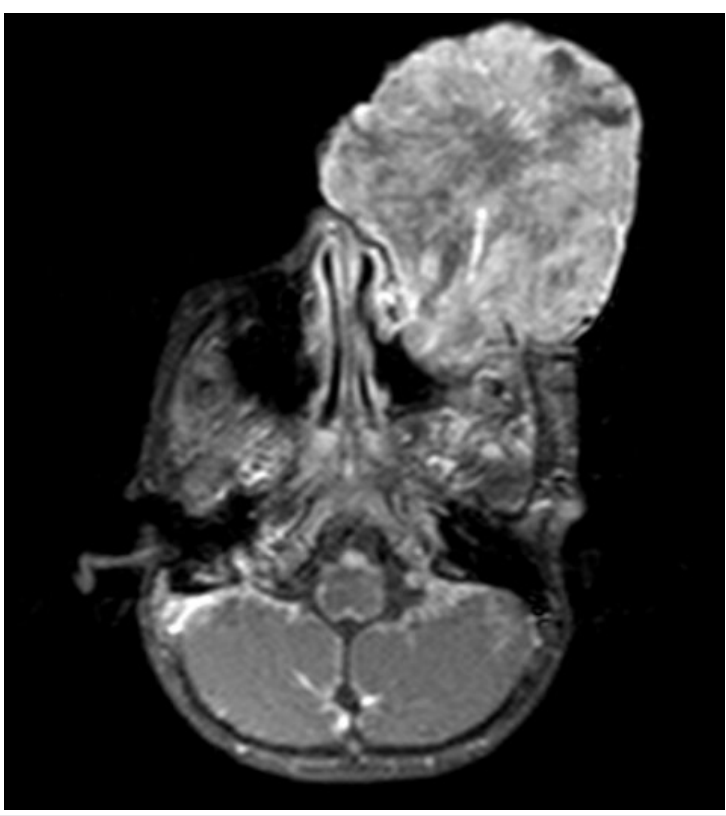

Figure 5. Axial T1-weighted fat-saturated magnetic resonance image with gadolinium showing moderate to marked enhancement in non-necrotic areas of the mass

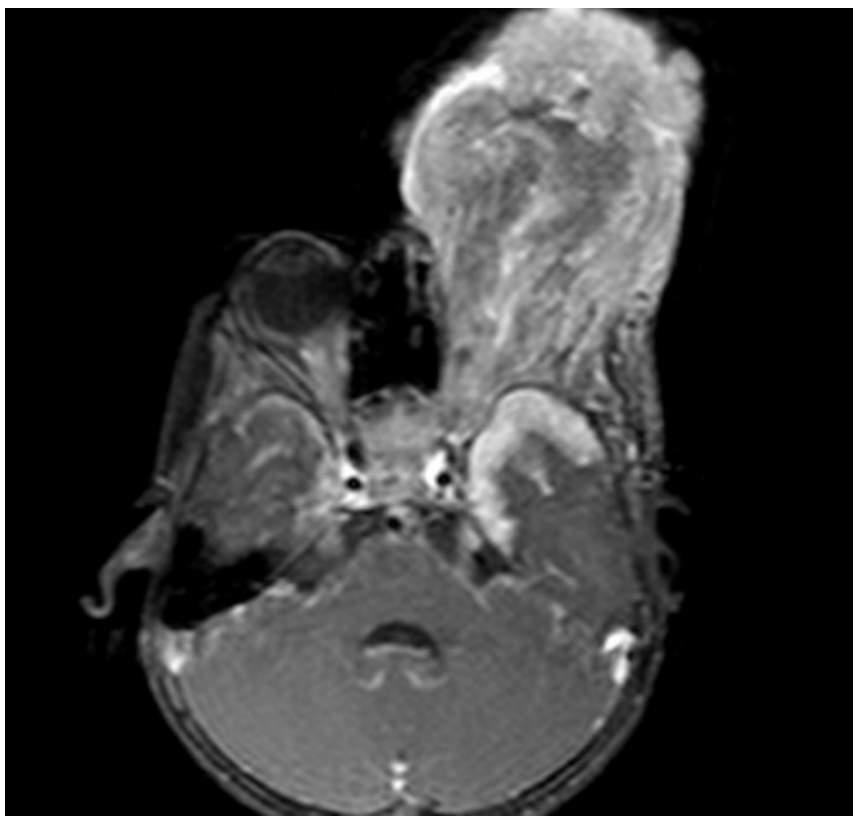

Figure 6. Axial T1-weighted fat-saturated magnetic resonance image with gadolinium showing metastatic leptomeningeal enhancement at the left temporal pole metastasis nor adrenal mass or paravertebral lesion suggesting orbital metastasis of neuroblastoma (NBL). Primary orbital NBL, primary orbital RBL, and orbital rhabdomyosarcoma (RMS) were considered in the differential diagnosis. Following left orbital enucleation, the diagnosis of orbital RBL was made by histopathological examination, which showed orderly proliferation of neoplastic cells with trabecular and insular pattern, occasionally presenting as Flexner-Wintersteiner type rosette formation (Figure 8). After surgery, the patient received radiotherapy and adjuvant chemotherapy. The patient was seen again in 2018 , but no recurrence was observed.

Informed consent has been taken from the family of our patient.

\section{DISCUSSION}

Primary orbital RBL is defined as an orbital extension of an intraocular RBL detected clinically or radiologically at the time of diagnosis (1). The term, microscopic orbital $R B L$, is used when an enucleated eye with intraocular RBL demonstrates ON invasion, evident scleral infiltration, or extrascleral extension on histopathological evaluation (6). Clinical presentation with an exuberant fungating orbital mass, as in the present case, is very rare and found in more advanced, untreated cases due to

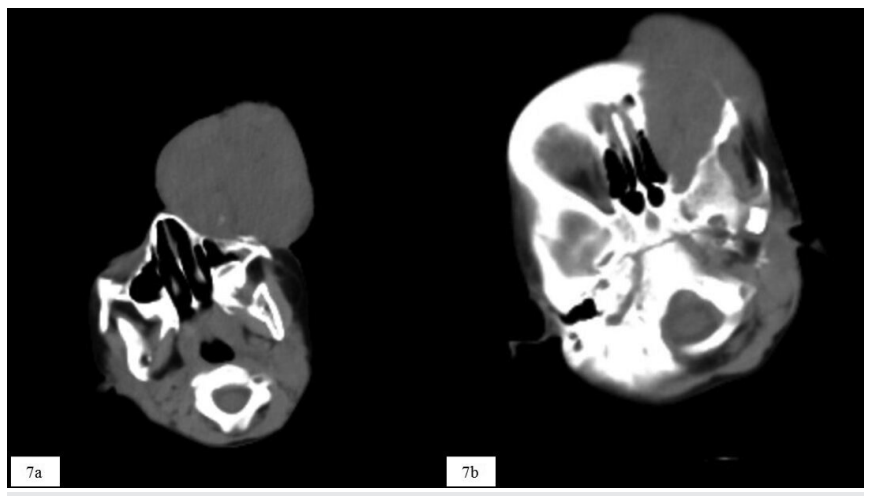

Figure 7. Axial computed tomography scan showing (a) the left orbital mass with some hemorrhagic foci but no calcification and (b) erosive destructive changes at the lateral orbital wall

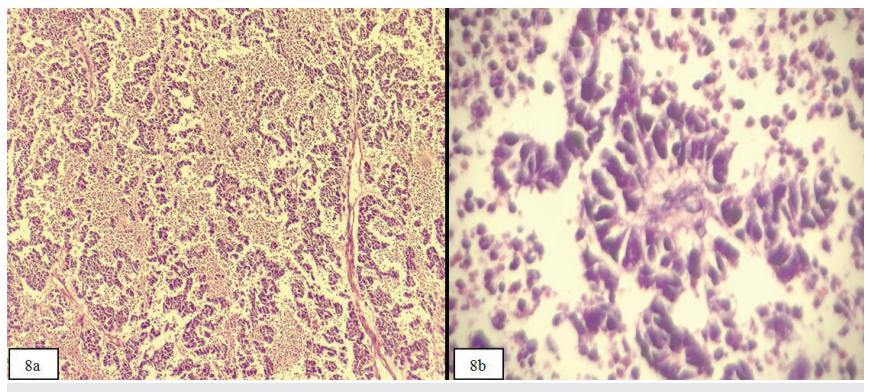

Figure 8. Histopathological examination showing (a) orderly proliferation of neoplastic cells with trabecular and insular patterns, occasionally forming rosettes (hematoxylin-eosin, x40). (b) Sections show a close-up view of a Flexner-Wintersteiner type rosette formation (hematoxylin-eosin, $x 400$ ) 
massive extraocular tumor spread through the $\mathrm{ON}$ or sclera. Both MRI and CT are used to assess the exact size and extent of the tumor. MRI, with its excellent soft tissue contrast, is the preferred imaging modality for the orbits and brain and for the evaluation of the tumor, vital orbital structures like the $\mathrm{ON}$, and intracranial involvements. In addition, MRI enables diffusion-weighted imaging (DWI) that is valuable in monitoring treatment response. CT is useful in the evaluation of adjacent bony structures and calcifications. Following contrast administration, moderate to marked enhancement is seen in non-necrotic parts of the tumor. The rapid growth of the tumor results in necrosis and heterogeneous enhancement. On DWI, the enhancing viable part of the tumor tissue shows restricted diffusion associated with apparent diffusion coefficients lower than those of non-viable necrotic tissues. However, since these imaging features are not specific for RBL, histopathological examination is needed for exact diagnosis. In the present case, the differential diagnosis included embryonal RMS, primary orbital NBL, and primary orbital RBL, all of which share similar histology that basically consists of small round blue cells. RMS is the most common primary orbital malignancy in children (7). Clinical presentation is a typical rapidly growing mass, often in the upper inner quadrant of the orbit (8). On histopathological examination, embryonal RMS, which accounts for the majority of orbital RMS cases, is differentiated by the presence of rhabdomyoblasts. By contrast, NBL is the most common metastatic orbital tumor in children, with the primary focus in the adrenal medulla or paravertebral sympathetic/ parasympathetic tissue of the abdomen and chest. In our patient, CT of the chest and abdomen did not reveal remarkable findings. Very rarely, NBL may be found as a primary orbital NBL without any systemic disease (9). Histologically, the undifferentiated part of the RBL demonstrates small blue cells with large hyperchromatic nuclei and scant cytoplasm. Homer Wright Pseudorosettes and Flexner-Wintersteiner Rosettes may be seen. Homer Wright Rosettes are differentiated tumor cells grouped around a central region containing neuropil and are therefore associated with tumors of neuronal origin and found in other tumors like pancreatic neuroendocrine tumors. On the contrary, FlexnerWintersteiner Rosette is a relatively unique characteristic of RBL. In our patient, occasional Flexner-Wintersteiner type rosette formation was found and the tumor was differentiated from NBL. Since the orbital extension of the intraocular RBL increases the risk for regional and systemic dissemination of the disease compared with patients without extraocular disease, detailed evaluation is mandatory (10). The most common metastatic dissemination of $\mathrm{RBL}$ occurs intracranially via the $\mathrm{ON}$ in which the subarachnoid space of the ON sheath is infiltrated by the tumor cells with dissemination into the cerebrospinal fluid (5). Therefore, MRI of the brain and spinal cord should be performed th the same time to assess leptomeningeal metastases in patients with extraocular extension of RBL. Brain MRI is also necessary to rule out trilateral RBL. In the present case, both supratentorial and infratentorial leptomeningeal metastases were found on the brain MRI at the time of presentation. In RBL, lymphatic spread to regional lymph nodes depends on the involvement of the conjunctiva or eyelids due to the absence of lymphatics in most of the globe and the orbit. Preauricular lymph nodes are most commonly affected in these patients (1). Therefore, they should be carefully palpated in clinical examination, and fine-needle aspiration biopsy should be done in case of suspicion. Metastasis to the lungs, bone, bone marrow, and liver may occur hematogenously. Skeletal metastases are best evaluated by bone scintigraphy or fluorodeoxyglucose positron emission tomography, because the highly hematopoietic bone marrow in infants makes it difficult to detect bone marrow involvement on MRI. On post-treatment follow-up, MRI is the preferred modality for the assessment of treatment response and detection of local and distant recurrences because of the increased long-term risk for malignancy in young patients who undergo repetitive CT. Currently, there are no standard treatment protocols for orbital RBL. Good long-term survival results have been reported with multimodal treatment, including high-dose chemotherapy, orbital enucleation, radiotherapy, and additional adjuvant chemotherapy over the last years. However, extraocular extension of RBL is still a major contributor to mortality, and patients have a poor prognosis (5).

\section{CONCLUSION}

Despite its rare occurrence in developed countries due to early diagnosis and treatment, primary orbital RBL is one of the major contributors to mortality. Early diagnosis and detailed radiological evaluation are essential to establish intracranial involvement and distant metastasis at the time of diagnosis. MRI, with its high tissue contrast and improved spatial resolution, is the mainstay in the diagnosis and follow-up of patients. Survival can be increased with early multimodal treatment and close radiological follow-up.

Informed Consent: Informed consent has been taken from the family of our patient.

Peer-review: Externally peer-reviewed.

Author Contributions: Concept - K.K., B.E., O.A.; Design - K.K., B.E., O.A.; Data Collection or Processing - K.K., B.E., O.A.; Analysis or Interpretation - K.K., B.E., O.A.; Literature Search - K.K., B.E., O.A.; Writing - K.K., B.E., O.A.

Conflict of Interest: There is no any conflict of interest among the authers.

Financial Disclosure: The authors declared that this study has received no financial support.

\section{REFERENCES}

1. Rauschecker AM, Patel CV, Yeom KW, Eisenhut CA, Gawande RS, O'Brien JM, et al. High resolution MRI of the orbit in patients with RBL. Radiographics 2012; 32: 1307-26.

2. Rao AA, Naheedy JH, Chen JY, Robbins SL, Ramkumar HL. A clinical update and radiologic review of pediatric orbital and ocular tumors. J Oncol 2013; 2013: 975908.

3. Kumar V, Abbas AK, Aster JC. Robbins and Cotran Pathologic Basis of Disease. 9. Philadelphia: Elsevier Saunders, 2014.

4. Robbins and Cotran pathologic basis of disease. Philadelphia, Pa. Elsevier Saunders, c2005.

5. Khelfaoui F, Validire P, Auperin A, Quintana E, Michon J, Pacquement $H$, et al. Histopathologic risk factors in retinoblastoma: a retrospective study of 172 patients treated in a single institution. Cancer 1996; 77: 1206-13. 
6. Honavar SG. Orbital Retinoblastoma. In: Singh AD, Damato BE, Pe'er J, Murphree AL, Perry J (Eds.), Clinical Ophtalmic Oncology, first ed., Saunders Elsevier, 2007.pp. 477-83.

7. Singh AP, Gupta AK, Mathur V, Barolia.DK. Embryonal rhabdomyosarcoma of the orbit in a child. Med J DY Patil Vidyapeeth 2018; 1: 276-8.

8. Weiss SW, Goldblum JR, Enzinger FM. Enzinger and Weiss's Soft Tissue Tumors. Mosby Inc, 2001.
9. Reznick A, Mukundan G, Sonu R, Lin LK.Pictorial Review of Histopathologically Diagnosed Orbital Tumors. Am J Ophtalmic Clin Trials 2019; 2: 1-9.

10. Abramson DH, Beaverson K, Sangani P. Screening for retinoblastoma: presenting signs as prognosticators of patient and ocular survival. Paediatrics 2003;112:1248-55. 